\title{
La finance comportementale vers une meilleure compréhension des marchés financiers
}

\section{TRID SABAH (Enseignant Chercheur)}

Laboratoire interdisciplinaire de recherche en économie, finance et management des organisations

Faculté des Sciences Juridiques, Economique et sociale -Fès

Université Sidi Mohamed Ben Abdellah Fès, Maroc

\section{EL GHMARI Omar (Doctorant)}

Laboratoire interdisciplinaire de recherche en économie, finance et management des organisations

Faculté des Sciences Juridiques, Economique et sociale -Fès

Université Sidi Mohamed Ben Abdellah Fès, Maroc

\section{LAREJ Issmail (Doctorant)}

Laboratoire études et recherche en management des organisations et des territoires

Faculté des Sciences Juridiques, Economique et sociale -Fès

Université Sidi Mohamed Ben Abdellah Fès, Maroc

\begin{abstract}
Résumé : La théorie de l'efficience des marchés financiers (EMH) est l'un des paradigmes fondamentaux en finance. En dépit de son apport considérable à la théorie économique et financière, elle se trouve depuis quelques années vivement contestées. Ses détracteurs mettent en avant l'incapacité de celle-ci à apporter des réponses pertinentes quant à certaines anomalies décelées sur les marchés financiers, notamment, le manque d'efficience, les effets calendriers, la sûr/sous-évaluation et la volatilité excessive, c'est pour cette raison que la finance comportementale s'est considérablement développée au cours des trente dernières années, amenant un nouveau regarde et une nouvelle réflexion sur l'analyse économique en général et la compréhension des marchés financiers en particulier.
\end{abstract}

Mots clés : efficience, rationalité, finance comportementale, biais comportementaux.

Digital Object Identifier (DOI): https://doi.org/10.52502/ijesm.v1i3.200

This work is licensed under a Creative Commons Attribution-NonCommercial-NoDerivatives 4.0 


\section{INTRODUCTION}

L'hypothèse d'efficience informationnelle représente les fondations de la finance moderne. Elle annonce que les cours boursiers englobent l'ensemble des informations relatives aux firmes et véhicule que le prix d'un titre converge à tout moment vers sa valeur fondamentale, grâce à l'usage d'ensembles d'informations pertinentes et exacts par des investisseurs rationnels, ce qui aboutit à créer cette égalité entre la vraie valeur du titre et son prix de vente sur le marché. Si l'offre et la demande ne sont heurtées par des données potentielles pertinentes, alors le cours boursier représente directement la valeur fondamentale du titre.

Toutefois, en présence d'investisseurs irrationnels, les erreurs repérées seraient corrigées par le mécanisme d'arbitrage qui aboutira au maintien de la valeur fondamentale. Pour les actions par exemple, la valeur fondamentale est égale à la valeur actualisée des dividendes futurs. Ce qui met en évidence l'obligation d'anticiper rationnellement les dividendes futurs à travers la recherche, l'analyse et l'exploitation des informations disponibles qui remplissent un rôle primordial et décisif dans la prise des choix. Ces investisseurs se focalisent à la pertinence de l'information publique circulant sur le marché, à leurs informations privées, à l'anticipation des informations privées détenues par les autres agents économiques et au calcul de la vitesse d'incorporation et d'intégration des données dans le cours des titres.

Cependant, cette théorie se trouve dans l'incapacité de donner des explications et des démonstrations convenables et adéquates à quelques anomalies repérées dernièrement sur les marchés financiers à savoir la sûr/sous-évaluation, l'excès de volatilités, les bulles de spéculations , les effets tailles et effets calendriers, alors force est de constater que la remise en doute de l'efficience de l'hypothèse d'efficience invalidera par la suite la notion de rationalité absolue des intervenants en marché financier, ou tout intervenant est dans la conquête de maximiser son profit quel que soit l'effort qui doit fournir afin de gérer les calculs analytiques et les tries d'informations utiles.

Ces contradictions remarquables de l'efficience durant ces trente dernières années a conduit des psychologues à s'introduire en milieu financier et déclarer que les décisions des individus sont éventuellement biaisées par les émotions les sensations, les heuristiques et l'état mental. KAHNEMAN et TVERSKY sont les fondateurs d'un courant récents qui impliquent à la fois finance et psychologie, ces deux auteurs soulignent le fait que les investisseurs sont la proie de plusieurs facteurs psychologiques qui influence directement et spontanément leurs interprétations, raisonnements et décisions d'où la naissance de la notion de rationalité limitée. L'étude des comportements humains en général et la psychologie en particulier a permis de concevoir des explications plausibles et convenables aux écarts constatés par rapport à l'hypothèse d'efficience des marchés. Cette cohésion entre la finance et d'autres sciences sociales a marqué la naissance de la finance comportementale qui introduit un renouvellement majeur au milieu financier.

Afin d'orienter notre présentation, nous allons opter pour la problématique suivante :

Dans quelle mesure la finance comportementale permet-elle une meilleure compréhension des marchés financiers? 
À partir de la problématique, on peut retenir les questions de recherche suivantes :

- Quels sont les fondements théoriques des marchés efficients et quelles sont leurs limites?

- Quel est l'intérêt d'une introduction de la finance comportementale dans le cadre de la compréhension des dysfonctionnements des marchés financiers?

Afin de mieux cerner notre présentation et de donner une certaine logique à notre étude, nous allons subdiviser notre tâche selon deux points, un premier traitant l'analyse fondamentale ainsi que les principales fissures de la théorie dominante et puis dans un deuxième, nous allons mettre en lumière l'avènement de la théorie comportementale ainsi que les indispensables biais et heuristiques et leurs explications présentées, ainsi que la proposition de quelque apports et limites de cette théorie.

\section{ANALYSE FONDAMENTALE ET PRINCIPALES FISSURES DE L'ÉDIFICE DOMINANT}

\subsection{La théorie d'efficience des marchés}

Nul ne peut énoncer l'hypothèse d'efficience des marchés sans évoquer le rôle prépondérant d'Eugène Fama pour la conception de ladite théorie de l'efficience ou HEM (l'hypothèse d'efficience du marché). En effet, c'est grâce à l'application des mathématiques probabilistes au milieu financier, durant les années 50 à 60 , ce qui a servi de socle de départ pour la modélisation financière et le développement immédiat des nouveaux outils de finance de marché.

Selon Fama $(1965)^{1}$, un marché est considéré comme informationnellement efficient si le prix d'un actif est égal à sa valeur fondamentale telle que l'on peut la déterminer sur la base de toute l'information disponible.

\section{L'importance de l'information:}

Selon FAMA la théorie des marchés efficients définit l'efficience d'un marché par son aptitude à réaliser ses fonctions. Un marché est donc efficient si les prix dans celui-ci constituent des fondements fiables pour ses acteurs afin d'agir. Ce qui met en évidence l'élément informationnel qui prendra alors une dimension incontournable dans cette hypothèse. D'une autre manière, la définition classique d'un marché efficient est celle d'un marché où les prix des actifs financiers cotés engendrent et prennent en considération toutes les informations disponibles donc aucune régulation ou réévaluation n'est possible pour le maintien de la juste valeur des titres à tout moment, elle considère que dans un marché suffisamment large où l'information se répand instantanément, prenant l'exemple du marché boursier où les opérateurs réagissent correctement et quasi immédiatement aux informations s'ils ont la capacité cognitive de les interpréter avec justesse. Ainsi, le prix d'un actif financier est à tout instant une estimation sans biais de sa valeur

\footnotetext{
${ }^{1}$ FAMA, E. (1965), « The behavior of stock-market prices », The Journal of Business, Vol. 38, No. 1 (Jan. 1965), pp.
} 34-105. 
intrinsèque. Il est par conséquent impossible de prévoir ses variations futures puisque tous les évènements connus ou anticipés sont déjà incorporés dans le prix actuel. ${ }^{2}$

Toutes fois l'efficience informationnelle est fondamentale pour le bon fonctionnement des marchés puisqu'elle permet de crédibiliser et attraire les investisseurs. Du coup, cette efficience est très fondamentale pour le développement des marchés financiers. C'est la raison pour laquelle toutes les autorités des marchés et places boursières cherchent à instaurer les fondements réglementaires et organisationnels pour aboutir à cette situation d'efficience informationnelle. Toutefois, la réalité des marchés fait que la transmission de l'information utile par le canal des prix n'est pas évidente puisque les investisseurs se trouvent heurtés par le manque d'information. Dans le cas d'un marché inefficient, les agents ne disposent pas de la valeur juste des actifs et sont en perpétuités à la recherche de toutes données utiles permettant l'évaluation convenable et adéquate pour la prévision des risques, des gains et des opportunités qui leur sont offertes par le marché. Dans cette première partie de notre article, nous allons établir le lien existant entre le concept de la rationalité des investisseurs et l'efficience des marchés.

\subsubsection{La rationalité du comportement des acteurs du marché :}

La deuxième acceptation du concept d'efficience des marchés financiers dépend tout d'abord de la rationalité du comportement des investisseurs. Selon cette acceptation, ainsi que le principe de valorisation, si le prix d'un actif financier reflète l'attente des revenus futurs qu'il génère, alors le marché des actifs financiers est efficace. On dit que le marché financier est efficace, car les prix des actifs cotés ne reflètent que les attentes des investisseurs quant à leurs revenus futurs. En effet, les investisseurs doivent non seulement comprendre correctement la valeur de base des actifs, mais également comprendre correctement le modèle réel. Par conséquent, il est nécessaire de disposer d'un inventaire d'informations suffisamment important pour analyser raisonnablement la tendance des prix afin d'obtenir les meilleures conditions requises. Dans le but de la fixation du prix, par la meilleure estimation possible de la valeur intrinsèque des titres, toutes en respectant le principe de rationalité financière qui confronte les traders à une problématique de maximisation du profit.

La recherche financière suit la trajectoire que suivent les économistes lorsqu'ils étudient le comportement des agents sur le marché des biens et services dans une économie. En effet, les économistes ont ressenti le besoin de déterminer le comportement des individus à travers l'étude des personnes dans leur environnement économique afin de pouvoir développer leurs théories. Afin de simuler le comportement des individus, ils ont modélisé sur la base de spécifications, dans le but de chercher à établir un équilibre dans la relation économique entre individus et entre économies, et ils ont introduit la rationalité dans le problème de l'utilisation de ressources rares pour répondre à la demande concept. Le but ultime de l'économie.

Dans la même logique, les financiers ont également assumé la rationalité des acteurs des marchés financiers afin de pouvoir développer des théories et modéliser les comportements observés sur ces marchés. Parmi ces théories, la théorie de l'efficience des marchés qui suppose la rationalité des

\footnotetext{
${ }^{2}$ Said AIT DANI \& Bouchra RADI , La controverse sur l'efficience des marchés financiers et l'émergence du paradigme comportemental ,Moroccan journal of business studies Vol1 Issue ${ }^{\circ} 1, \mathrm{p} 154$.
} 
agents tant dans leurs comportements que dans leurs anticipations, ce qui revient au fait que la rationalité est extrêmement nécessaire à l'efficience.

\subsubsection{Définition du concept « rationalité »}

Selon la définition d'ALLAIS (1953) ${ }^{3}$, « un homme est réputé rationnel lorsqu'il poursuit des fins cohérentes entre elles et qu'il emploie des moyens appropriés aux fins poursuivies ». Sur un plan plus général, le « rationnel » désigne ce qui converge vers la raison ou ce qui relève de la raison et « l'irrationnel » désigne ce qui n'est pas adéquat avec la raison ou au domaine de la raison. Allant dans ce sens, les psychologues DROZDA-SENKOWSKA et al. (1995) définissent un homme rationnel comme un homme raisonnable, un homme sain d'esprit (à l'abri de la folie) et un homme doué de la raison (au sens de la faculté à raisonner et à juger) ${ }^{4}$.

La rationalité a généralement trois significations, qui tendent vers une philosophie qu'un concept psychologique. Ces trois significations, même si elles sont complètement différentes, sont toujours étroitement liées les unes aux autres :

- Rationnel signifie justifier, démontré, approuvé : fondé sur une raison incontestable, d'où nul ne peut la mettre en doute.

-Rationnel signifie aussi critique et investigation, qui ne se laisse pas emprisonner par les évidences apparentes et les choix antérieurs.

-Rationnel est compris aussi comme efficace au sens de maximiser les rendements espérés.

Les deux premiers sens reflètent mieux les définitions qui intéressent les psychologues sociaux, tandis que le second sens est adopté par les économistes et les financiers. En effet, d'un point de vue économique, tout individu capable de définir clairement ses besoins et d'optimiser l'utilisation des ressources tout en choisissant le service le meilleur ou le plus adapté pour répondre directement à ce besoin est considéré comme rationnel.

Cependant il existe plusieurs formes de rationalités on recense :

-La rationalité instrumentale ou classique : D'après ALLAIS (1953), les agents sont distingués en fonction des préférences ou des objectifs qu'ils cherchent à atteindre, en tenant compte des contraintes qui limite la portée de leurs choix possibles. Il s'agit donc de la Rationalité instrumentale qui représente l'adéquation entre les moyens (revenu) et les fins souhaitées.

-La rationalité cognitive : les capacités cognitives de l'individu ont également conduit au développement d'autres concepts rationnels basés sur la modération de la rationalité classique. WALLISER (1982) ${ }^{5}$ a introduit le concept de rationalité cognitive pour considérer la correspondance entre l'information détenue par l'agent et la représentation (les croyances

\footnotetext{
${ }^{3}$ Allais, M. «Le Comportement de l'Homme Rationnel devant le Risque : Critique des Postulats et Axiomes de l'Ecole Américaine », Econometrica, Vol. 21, No. 4 (Oct., 1953), pp. 503-546.

${ }^{4}$ DROZDA-SENKOWSKA, E. (1995) "La rationalité des inférences : quelques remarques sur la pertinence de ce concept dans les analyses psychosociales", Les Cahiers Internationaux de Psychologie Sociale, pp. 9-26.

${ }^{5}$ WALLISER, B., (1982), "Equilibres et anticipations", Revue Economique, Vol. 33, No. 4 (Jul., 1982), pp. 594-638.
} 
représentatives varient d'un agent à l'autre). Par conséquent, la rationalité cognitive met l'accent sur le fait qu'il peut y avoir des différences entre l'environnement réel et l'environnement perçu, reflétant ainsi si les informations détenues sont conformes à l'environnement.

- La rationalité limitée ou procédurale : cette notion est fondée principalement par SIMON $(1987)^{6}$, il met en exergue que les individus ne peuvent avoir que des contraintes imparfaites et des choix possibles. Herbert Simon a souligné que face à des individus dont l'information est imparfaite, les choix qu'ils font ne sont pas optimaux, mais tentent simplement de réaliser certains désirs ou exigences. La raison n'est plus absolue et objective, mais pleine de subjectivité et de relativité. La raison est avant tout procédurale, elle dépend donc dans une large mesure de l'organisation dans laquelle l'individu agit.

\subsection{Les conditions et formes des marchés efficients}

\subsubsection{Les conditions d'un marché efficient}

Dans un marché efficace, la concurrence entre les investisseurs est si féroce que le prix s'adaptera immédiatement aux nouvelles informations. À chaque instant, le prix de négociation des instruments financiers dépend des rendements ainsi que des risques appropriés. Cependant Fama (1965) souligne que, sur un marché efficient, à tout instant le prix effectif d'un titre sera une bonne estimation de sa valeur fondamentale.

La théorie d'efficience est fondée principalement par deux hypothèses ${ }^{7} \mathrm{de}$ bases inspirées généralement par la pensée néoclassique, qui implique d'une part la rationalité des acteurs du marché et d'autre part la concurrence pure et parfaite, cependant afin de distinguer un marché efficient, il doit valider les conditions suivantes :

Les intervenants sont rationnels : les investisseurs disposent pleinement et momentanément de toutes informations utiles à la prise de décision, ils réagissent de façon convenable aux données qu'ils reçoivent, toute fois inspirée par la maximisation du profit espéré , cette théorie met en évidence que les acteurs du marché sont parfaitement capables de se comporter justement quelle que soit la nature de l'information qui circule et doivent maximiser leurs gains potentiels pour n'importe quel niveau donné de risque, abstraction faite de leurs capacités cognitives qui peut heurtées à tout moment leurs volontés décisionnelles .

La concurrence est pure et parfaite : Le marché englobe autant d'acheteurs que de vendeurs parfaitement informés et sans être impacté par les frais de transactions.

1. La transparence de l'information: cette dernière doit circuler librement, fournit gratuitement, disponible à tout moment et simultanément pour tous les opérateurs, ce qui leur permet de la traiter en temps réel. Abstraction faite de toutes informations privilégiées. (Cas des délits d'initiés).

\footnotetext{
${ }^{6}$ SIMON, H.A., (1987), « Making management decisions: The role of intuition and emotion », The Academy of Management Executive, (1987-1989), Vol. 1, No. 1 (Feb., 1987), pp. 57-64.

${ }^{7}$ THAMI KABBAJ ,2007 'investir sans criser, bourse : saisir les opportunités “' Éditions d'Organisation ,pp :2123
} 
2. L'atomicité ou la présence de nombreux intervenants sur le marché : La théorie de l'efficience des marchés justifie ha Présence d'une multitude de spéculateurs qui favorisent la liquidité du marché. Ainsi nul ne peut influencer les cours du marché, puisque tous les acheteurs et vendeurs sont de taille atome.

3. L'absence de coûts de transactions et d'impôt de bourse: dans le cas de l'existence de barrières à l'entré ou à la sortis les agents économiques peuvent hésiter à investir et à désinvestir puisque les coûts de transactions ou les taxes boursières grèvent et pénalise leurs gains potentiels réalisables.

Selon les économistes, un marché efficace peut optimiser l'allocation des ressources, les prix des actifs cotés fournissent des informations précieuses aux bailleurs de fonds, de sorte que les ressources financières peuvent être allouées de manière optimale au moindre coût. Pour la théorie néoclassique, le prix est un signal. Il fournit toutes les informations nécessaires aux agents économiques pour prendre des décisions. Les prix proposés par les marchés financiers permettent aux investisseurs d'appréhender les axes de croissance et donc d'y investir. Pour cette approche, il est important que les prix reflètent les réalités économiques, non fixés par l'État ou d'autres institutions.

\subsubsection{Les différentes formes de l'efficience des marchés financiers}

Selon la définition de Fama (1965), caractérisée par sa généralité ainsi que la difficulté de vérification de cette dites notion d'efficience par un test unique, Fama $(1970,1991)^{8}$ se trouvait dans l'obligation de la subdiviser selon trois niveaux à savoir : la forme faible (« weak form »), la forme semi- forte («semi-strong form ») et la forme forte («strong form»). À cet effet on énonce quelques études empiriques, dans le but de vérifier ces différentes formes d'efficiences face à la prévisibilité des cours pour chacune des illustrations citées.

La forme faible d'efficience : en se basant sur la forme faible d'efficience, le cours d'un actif se focalise généralement sur son prix historique ou en évoque la notion «nul ne peut battre le marché » tout en se concentrant sur les prix anciens, cependant l'appellation de ce type d'efficience est instaurée à travers l'archive des prix passés, qu'on peut qualifier comme information abondante et disponible momentanément pour tous les acteurs du marché, d'une part nul ne peut bénéficier de cette donnée due à sa propagation au sein du marché, d'une autre part plusieurs investisseurs conduisent une étude chronologique des prix passés et volumes des transactions, afin de repérer la tendance à court terme et dégager des profits or l'hypothèse de la marche aléatoire se fonde sur l'indépendance des mouvements .

La forme semi-forte d'efficience : La forme semi-forte de la théorie de l'efficacité suppose que toutes les informations disponibles liées aux actifs financiers sont également engendrées dans le prix de ce titre au même moment où ces informations sont rendues publiques. Elles peuvent inclure toutes Informations sur la société émettrice, telles que rapports annuels, annonces-Bénéfices, distribution d'actions gratuites, informations fournies par la presse, etc. L'objet est de tester si le prix peut être ajusté rapidement pour s'adapter à ces informations.

\footnotetext{
${ }^{8}$ FAMA, E., (1991), « Efficient Capital Markets: II », The Journal of Finance, pp. 1575-1617
} 
L'importance est de vérifier si les prix annoncés se corrèlent immédiatement aux multiples informations perçues par le marché ainsi on met en lumières la capacité d'ajustement des résultats par le marché. Donc selon l'optique de FAMA on peut mettre en relief que si le marché est efficient selon le niveau semi-fort l'analyse fondamentale qui permet de sélectionner les actifs sous-évalués (à acheter) et les actifs surévalués (à vendre) est insignifiante.

La forme forte d'efficience : selon cette forme d'efficience les prix engendrent non seulement les informations divulguées publiquement elle comprend aussi les donnés détenues par les initiés de l'entreprise, c'est-à-dire toutes les informations publiques et privées ce qui est très délicat à vérifier empiriquement due aux caractères confidentiels des informations.

Le tableau suivant récapitule les différentes formes d'efficience informationnelle du marché financier avec des exemples :

Tableau 1. Formes d'efficience informationnelle des marchés financiers

\begin{tabular}{|c|c|c|c|}
\hline & \multicolumn{3}{|c|}{ Formes d'efficience informationnelle des marchés financiers } \\
\hline & Forme faible & Forme semi-forte & Forme forte \\
\hline $\begin{array}{l}\text { L'information reflétée } \\
\text { par les cours }\end{array}$ & $\begin{array}{l}\text { Les informations déjà } \\
\text { connues et publiées } \\
\text { dans le passé }\end{array}$ & $\begin{array}{l}\text { Toute l'information } \\
\text { publique }\end{array}$ & $\begin{array}{l}\text { Les informations } \\
\text { anticipées (non encore } \\
\text { publiées) }\end{array}$ \\
\hline $\begin{array}{l}\text { Exemples } \\
\text { d'informations }\end{array}$ & $\begin{array}{l}\text { Comptes publiés les } \\
\text { années passées, des } \\
\text { séries de taux } \\
\text { d'intérêt... }\end{array}$ & $\begin{array}{l}\text { Variation de taux } \\
\text { d'intérêt, publication } \\
\text { de comptes ou } \\
\text { annonce de résultat. }\end{array}$ & $\begin{array}{ll}\begin{array}{l}\text { Initiés } \\
\text { performances, }\end{array} & \text { et } \\
\text { analysent } & \text { des } \\
\text { professionnels } & \end{array}$ \\
\hline $\begin{array}{l}\text { L'information } \\
\text { permettant un gain }\end{array}$ & $\begin{array}{l}\text { Les informations sur } \\
\text { le présent et sur le } \\
\text { futur }\end{array}$ & $\begin{array}{ll}\text { Les } & \text { informations } \\
\text { privées } & \end{array}$ & $\begin{array}{l}\text { L'information } \\
\text { disponible n'enrichit } \\
\text { personne }\end{array}$ \\
\hline
\end{tabular}

\subsection{Principales fissures du paradigme dominant et justificatif de la finance comportementale}

\subsubsection{Le manque d'efficience des marchés}

La finance classique repose notamment sur l'hypothèse de marchés efficients, ce que remet en question la finance comportementale appuyée par des défis convaincants. Dans la théorie classique, le prix des actifs financiers reflète l'ensemble des données publiques disponibles. C'est un monde parfait, le prix est l'indicateur parfait de la valeur. Fama pense que les données antérieures ont été incorporées au cours. Le passé ne diffuse aucun signe de l'avenir, on parle de

\footnotetext{
${ }^{9}$ RAHAOUI, R., (2007), « L'efficience informationnelle du marché financier Marocain : L'impact d'un profit warning sur les cours boursiers », Mémoire Online, http://www.memoireonline.com/02/09/1969/m_Lefficienceinformationnelle-du-marchefinancier-Marocain-Limpact-dun-profit-warning-sur-les-cou10.html (consulté le 04/04/2021).
} 
"random walk" ou marche aléatoire, ce qui signifie que le cours de l'action est aléatoire à court terme si on considère l'absence de données pertinentes ou anticipations de résultats.

Dans le même contexte, la théorie classique de la finance s'appuie sur les acteurs de marché et c'est grâce à leurs rationalités que le marché aboutit à l'efficience. Une des répercussions immédiates est la convergence entre le prix d'une action et sa valeur fondamentale, la valeur intrinsèque de l'action d'une société est la somme actualisée des flux futurs prévisionnels générés par cette dernière.

Valeur fondamentale $=\operatorname{Flux}(1) /(1+r)^{1}+\operatorname{Flux}(2) /(1+r)^{2}+\ldots+\operatorname{Flux}(t) /(1+r)^{t}$

Avec $\mathrm{r}=$ taux d'actualisation

Flux(t), les cash-flows générés par la société à un instant futur t.

Théoriquement, dès qu'une survalorisation ou une sous-valorisation s'installe entre la valeur de marché et la valeur fondamentale, on a affaire à l'arbitrage sans risque pour les agents rationnels.

\section{Figure 1 : Sur / sous valorisation et valeur fondamentale}

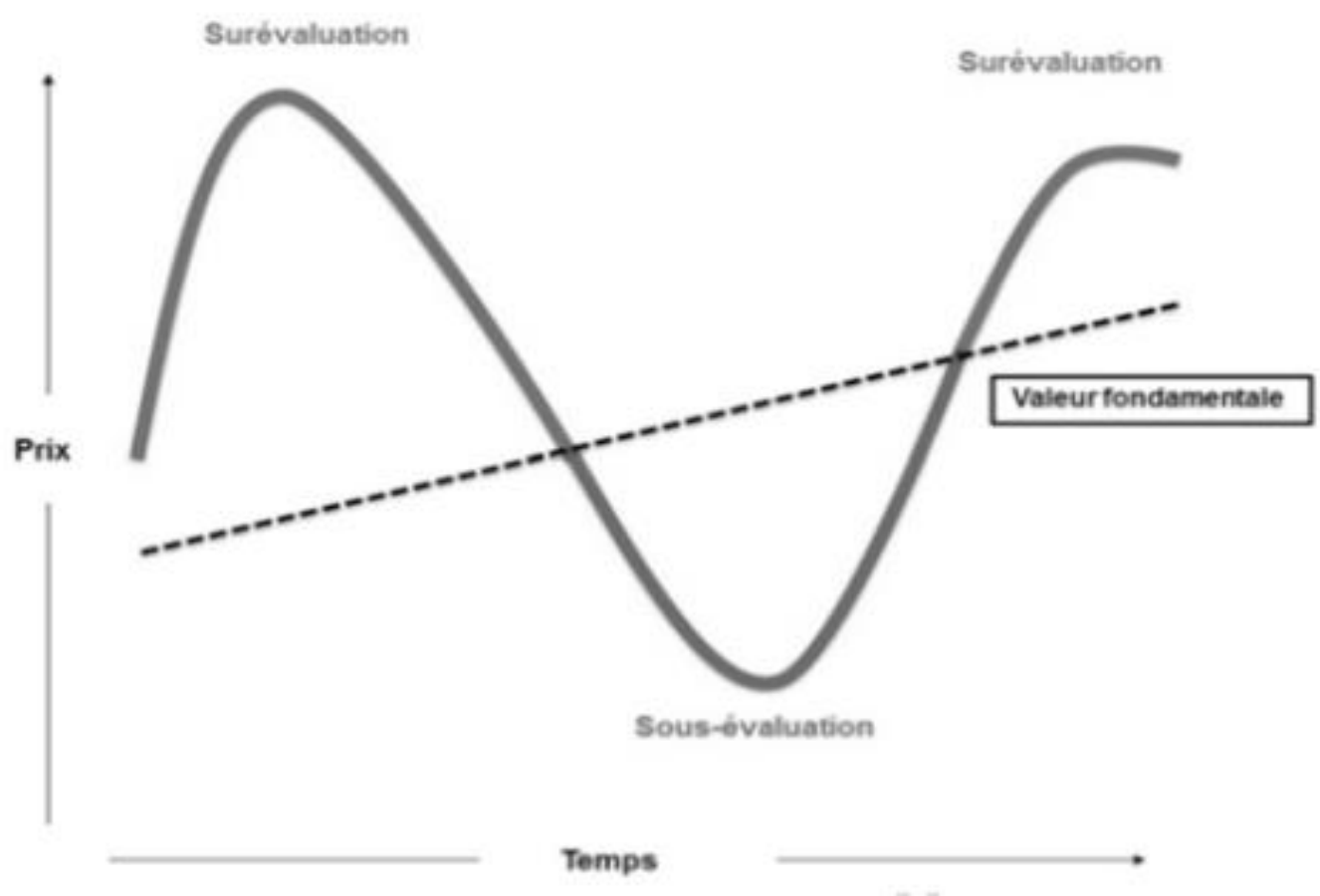

Source : Yannick Coulon $(2015)^{10}$

Cet écart repéré disparaît grâce aux intervenants et des acteurs de marché qui en tirent bénéfices. La surcote d'une action par rapport à sa valeur fondamentale signifie un gain instantané qui stimulera les ventes et ramènera le prix de l'action au niveau de sa valeur fondamentale. Alors

\footnotetext{
${ }^{10}$ YANNICK COULON, «Guide pratique de la finance comportementale», Gualino éditeur, Lextenso éditions 2015,p
} 14 
qu'une décote déclenche des achats, qui réduiront eux aussi les prix au niveau de leurs valeurs fondamentales. Ce mécanisme élimine donc spontanément les différentiels de valorisation sur les marchés.

La finance comportementale remet ce dogme en question, car elle considère que : l'arbitrage est lié à un risqué, qui se traduit par la confrontation à deux obstacles significatifs :

-La présence des agents irrationnels qui se basent sur des fausses informations, rumeurs ou noises traders

-Le retour vers la valeur fondamentale n'est pas immédiat sachant que cette opération nécessite une performance absolue

\subsubsection{L'excès de volatilité et des volumes de transaction}

Dans une optique théorique de marché efficient, la volatilité des prix des actifs devrait évoluer dans des marges raisonnables, ce qui a été mis en question par Gervais et Odean (2001) ${ }^{11}$ qui mettent en évidence que les prix des actions se caractérisent par une volatilité plus excessive que prévoit l'hypothèse d'efficience. Ces derniers annoncent que la volatilité des prix relativement aux fondamentaux est élevée. Les résultats de cette étude sont en concordance avec les recherches de Shiller (1981) ${ }^{12}$, l'un des premiers auteurs à repérer l'existence d'une volatilité excessive sur les marchés financiers. Il a comparé les volatilités des cours et des fondamentaux. Le test consiste à comparer l'évolution des prix réellement observés sur le marché à celle des prix optimaux déterminés séparément, qu'on peut déterminer en fonction des dividendes effectivement collectés ce qui est malheureusement différent de la valeur fondamentale calculée du titre.

Alors que si le marché était efficient, les deux valeurs devraient exhiber des dynamiques très proches. Donc il est indispensable de déclarer que les prix calculés statistiquement sont plus stables et lisses que ceux repérés au niveau du marché, ce qui remet en cause directement l'hypothèse d'efficience informationnelle. Cette volatilité excessive des prix est due à la présence d'investisseurs irrationnels qui agissent à des données qui n'ont pas de liaison avec les fondamentaux notamment leurs croyances et perceptions, ces erreurs d'évaluations seront difficilement corrigées par les investisseurs rationnels.

\subsubsection{L'effet cycle}

L'une des premières anomalies visionnées au niveau des marches est celle repérée en fin de cycle, ces déviations représentent des poches d'inefficiences temporaires. On cite l'anomalie fin de semaine et fin d'année.

- L'anomalie fin de semaine présentée par Osborne $(1962)^{13}$ il met en lumière que quelques titres ont une moyenne négative le lundi et positive tout au long de la semaine, ce qui a été confirmé

${ }^{11}$ GERVAIS S., ODEAN T., (2001), "Learning to be overconfident". Review of Financial Studies, 14, pp. 1-27

${ }^{12}$ SHILLER R. J., (1981),"Do stock prices move too much to be justified by subsequent changes in dividends?". American Economic Review, 71, pp. 421-498.

${ }^{13}$ OSBORNE M., (1962), "Periodic Structure in the Brownian Motion of Stock Prices". Operations Research, 10, pp. 345-379. 
par d'autres auteurs, Venezia et Shapira (2007) ${ }^{14}$ qui démontre que les rendements pendant les jours qui suivent le weekend sont moins intéressants que ceux des autres jours, c'est pour cette raison que les professionnels ont tendance à réduire les transactions en début de semaine.

- L'anomalie fin d'année appelée aussi effet janvier introduite par Rozeff et kinney(1976) ${ }^{15}$ qui souligne que les actions enregistrent des rentabilités supérieures en janvier et particulièrement les deux premières semaines, la justification de ce phénomène est l'incident fiscal. En fait, les moins-values dégagées transfèrent les investisseurs à réaliser leurs pertes en fin d'année afin de minorer leur revenu imposable. Une justification de cet incident a été avancée par la finance comportementale, c'est la perception des investisseurs que la fin d'année est le moment du bilan et le début d'année comme un nouveau départ, cette anomalie est relative à la comptabilité mentale.

Plusieurs anomalies relatives aux caractéristiques des firmes ont été décelées, ce sont des irrégularités dont la justification ne réside pas au niveau des modèles fondamentaux fondés sur l'hypothèse d'efficience, ces modèles sont le modèle de marché et le modèle d'évaluation des actifs financiers.

\section{L'INSTAURATION DE LA DIMENSION COMPORTEMENTALE UN LEVIER DE MAITRISE DES MARCHÉS FINANCIERS}

\subsection{Avènement de la finance comportementale}

A contrario avec les modèles théoriques qui se focalise sur un seul investisseur représentatif, De Long et al. (1990) ${ }^{16}$ ont mis en lumière l'existence de deux types d'investisseurs : d'une part les investisseurs rationnels et d'autre part les investisseurs irrationnels qu'on peut qualifier de bruiteurs («noise traders »). On les définit comme des investisseurs qui se comportent sur la base de croyances erronées. Ils opèrent sur la base du comportement des autres investisseurs et écartent les prix des titres de leurs valeurs fondamentales. Selon l'hypothèse d'efficience informationnelle, les investisseurs rationnels saisissent toutes opportunités d'arbitrage afin de réaliser des profits.

La recherche psychologique montre que le comportement réel d'un individu n'est pas interprété par les hypothèses de la théorie standard. Tversky et Kahneman (1974) ${ }^{17}$ ont révélé que les individus choisissent des raccourcis dans le raisonnement, ce qui conduit à des décisions biaisées.

En effet, au début des années 2000 plusieurs études ont été menées par des psychologues sont repris par les chercheurs en finance à l'instar des travaux de Tversky et Kahneman. Le point

\footnotetext{
${ }^{14}$ VENEZIA I., SHAPIRA Z., (2007), "On the behavioral differences between professional and amateur investors after the weekend". Journal of Banking \& Finance, 31, pp. 1417- 1426

${ }^{15}$ ROZEFF M.S., KINNEY W.R., (1976), "Capital Market Seasonality: The Case of Stock Returns". Journal of Financial Economics, 3, pp. 379-402.

${ }^{16}$ DE LONG, J. B., SHLEIFER, A., SUMMERS, L. H., WALDMANN, R. J., (1990), "Noise trader risk in financial markets". Journal of Political Economy, 98, pp. 703-738.

17 TVERSKY A., KAHNEMAN D., (1974), "Judgment under uncertainty: Heuristics and biases". Science, 185, pp. $1124-1131$.
} 
de départ de la finance comportementale est de tester la rationalité des agents ainsi que les axiomes associés, les résultats et constats obtenus sont les suivants :

Les axiomes d'indépendance et de transitivité sont souvent dépassés par des décisions individuelles ;

Les investisseurs ont un comportement moutonnier ;

Les investisseurs ne suivent pas la règle de BAYES ;

Les investisseurs sont surconfiants ;

Les investisseurs simplifient les choix complexes à l'aide de règles et de raccourcis et raccourcis nommés heuristiques

Les heuristiques décrivent les principes et des méthodes qui permettent aux agents de porter facilement et spontanément des jugements ou des évaluations raisonnables. Il s'agit d'un processus de jugement, sans aucune méthode d'analyse délibérée ni contrainte de quantification ou de traitement, ce concept est crucial en finance comportementale, car il permet de comprendre comment les opérateurs s'écartent de la rationalité. Tous ces constats ont conduit les chercheurs à rejeter l'hypothèse de rationalité des agents et celle de l'efficacité du marché financier.

\subsection{Les principaux biais et heuristiques de la finance comportementale}

Plus généralement, la finance comportementale tentera d'expliquer les anomalies de marche à partir de l'étude du comportement humain. Puisque c'est lors de la prise de décision qui est parfois complexe, que les individus prennent souvent des raccourcis mentaux plutôt que de s'engager dans des investigations et des traitements analytiques très longs. On s'intéresse particulièrement aux différentes heuristiques suivantes :

- Biais de familiarité

Lorsqu'une personne préfère une option à une autre, un «biais de familiarité » apparaît, car il lui est plus familier. Après avoir mené différents questionnaires, TVERSKY a montré qu'entre deux paris offrant la même probabilité de succès, les individus choisiront celui qu'ils connaissent le mieux. L'auteur prouve également que même si la probabilité de succès est faible, quelques personnes parieront toujours plus familières.

- Biais émotionnels

Si les émotions peuvent être utiles au processus de prise de décision en le simplifiant, elles limitent également la capacité des investisseurs à analyser raisonnablement et efficacement. De manière générale, les investisseurs vivent différents états émotionnels (fierté, joie, angoisse, colère, euphorie, peur ...) et c'est l'humeur qui conduit à des actions prédéterminées. Les préjugés émotionnels sont également liés aux croyances, ils font l'objet de nombreuses études et peuvent influer sur les jugements des investisseurs. 
- Biais de cadrage

Appelé aussi "'effet de présentation", introduit par KAHNEMAN et TVERSKY grâce aux travaux d'économie comportementale, il peut être défini : les opérateurs n'ont pas le même comportement de décision face à un même problème présenté en des termes différents.

- Comportement suiveur

Sur les marchés financiers, l'imitation semble être la source des comportements de groupe qui conduisent à des écarts par rapport aux prix de la valeur fondamentale. La finance comportementale tend à justifier ce phénomène par des investisseurs ayant une rationalité limitée et ne peuvent pas gérer toutes les informations disponibles. Ensuite, ces investisseurs vont se trouver dans l'obligation de suivre les autres agents.

- Effet de disposition

L'effet de disposition est la tendance à vendre prématurément les titres gagnants et de maintenir trop longtemps les titres perdants. À première vue, l'effet peut sembler insignifiant, mais beaucoup d'études montrent souvent que cet effet est catastrophique en termes de rendement du portefeuille.

\subsection{Apports et limites de la finance comportementale.}

La finance comportementale permet de proposer des hypothèses plus réalistes pour comprendre les comportements financiers. Chaque trait comportemental, explique un phénomène observé sur le marché, cela était auparavant associé au paradigme classique qui malheureusement ne pouvait donner une explication convenable ce qui remet en question l'efficacité et le rejet du modèle de rationalité substantiel ce qui constitue une véritable révolution, faisant de la finance l'un des domaines les plus féconds. En effet, à la recherche d'une explication d'anomalie on observe l'opportunité pour les chercheurs de s'intéresser aux sciences cognitives et sociales, tout en renouvelant les facteurs qui influencent la prise de décision humaine.

Si la finance comportementale se focalise sur les aspects cognitifs et psychologiques d'autre courant ont opté pour des variables sociaux, politiques ou culturels, prenant l'exemple d'AKERLOF et SHILLER (2013) ${ }^{18}$, inspirés du modèle keynésien d'esprits animaux qui mettent en lumière les modèles populaires, $d$ 'autres auteurs expliquent quelques phénomènes par la force des institutions et des organisations.

L'une des critiques les plus démarquer au milieu scientifique de la finance comportementale est la conservation de l'hypothèse d'individualisme ainsi elle se concentre sur un seul et unique décideur en soulignant que la majorité des auteurs comportementalistes proposent des modèles micro-économiques ainsi l'incapacité à fournir un modèle général, FAMA $(1998)^{19}$ qui répond aux critiques d'efficience, lorsque l'auteur a remarqué que les résultats empiriques invalident

\footnotetext{
${ }^{18}$ AKERLOF G. et SHILLER R., 2013, Les Esprits animaux. Comment les forces psychologiques mènent la finance et l'économie, Paris, Flammario,p293

${ }^{19}$ FAMA E., 1998, Market Efficiency, Long-Term Returns and Behavioural Finance, Journal of Financial Economics, 49, 3, pp283-306.
} 
l'argument de l'efficacité, il a souligné que les manifestants n'ont pas non plus été en mesure de fournir un modèle général pour expliquer l'inefficacité des cours. Par conséquent, il souligne les réelles limites des méthodes de finance comportementale. Sa nature inductive et réaliste a conduit à la découverte de facteurs qui expliquent le comportement financier sans la capacité a proposé un modèle qui englobe généralement l'impact de tous ces facteurs sur la formation des prix. Absence d'une telle théorie permettent de remettre en cause la contestation scientifique du modèle de la finance comportementale.

\section{CONCLUSION}

Pour conclure, et dans la finalité d'assurer une compréhension aisée des marchés financiers, nul ne peut nier la place considérable que prend la théorie de l'efficience fondée sur la théorie d'équilibre concurrentiel qui met en exergue l'atomicité des intervenants, la contrainte de maximisation des profits, la transparence, la mobilité des informations et le retour vers la valeur fondamentale cette théorie qui se focalise sur le concept du marché imbattable et la marche aléatoire des marchés.

Cette situation théorique typique et adéquate au bon fonctionnement des parties prenantes au sein des marchés rend sa validation empirique très délicate sachant que malheureusement le concept de la rationalité des agents reste étroitement lié à l'hypothèse d'efficience et la base des modèles d'évaluations des actifs financiers (MEDAF) qui ont été remis en cause par quelques économistes et financiers vu que la rationalité telle qu'a annoncé notre théorie de bases reste une situation fictive et la présence de biais ou heuristiques reste primordiale pour la majorité des cas ou les investisseurs sont confrontés à des situations délicates ou nécessitant un traitement analytique compliqué . Ces raccourcis décisionnels brisent le concept de rationalité traditionnel et souligne le fait d'existence d'agents irrationnels ce qui a donné lieu à l'émergence de la finance comportementale qui donne une approche séduisante et remarquable à l'explication des anomalies repérées dans le marché en adoptant des méthodes et voies de recherches non exploitées par l'approche normative.

La finance comportementale, ayant pour finalité l'étude des comportements réels des investisseurs au niveau des marchés financiers, en se basant sur la psychologie sociale et cognitive. Ces disciplines tentent de répertorier et d'expliquer les écarts entre le comportement réel et celui adéquat à partir des informations disponibles, pour vérifier leur validité ou leur infaillibilité, ainsi que la mise en marche de ces informations tout en étudiant les différents biais et erreurs, dans le traitement de l'information, elle met aussi en évidence les éventuels défauts et anomalies dans la conception de la réalité et permet distinguer la rationalité limitée.

La théorie de la finance comportementale assure une meilleure compréhension du fonctionnement réel des marchés financiers par le biais de l'utilisation des approches psychologiques et sociologiques donnant une approche séduisante, simple, réelle et concevable pour l'explication des évènements existants et pouvant exister dans le milieu financier, toutes fois cette théorie reste mal exploité et désister par la théorie financière moderne.

\section{BIBLIOGRAPHIES}


[1] AKERLOF G. et SHILLER R., 2013, Les Esprits animaux. Comment les forces psychologiques mènent la finance et l'économie, Paris, Flammario, p293.

[2] ALLAIS, M. « Le Comportement de l'Homme Rationnel devant le Risque : Critique des Postulats et Axiomes de l'Ecole Américaine », Econometrica, Vol. 21, No. 4 (Oct., 1953), pp. 503-546.

[3] DE LONG, J. B., SHLEIFER, A., SUMMERS, L. H., WALDMANN, R. J., (1990), "Noise trader risk in financial markets". Journal of Political Economy, 98, pp. 703-738.

[4] DROZDA-SENKOWSKA, E. (1995) "La rationalité des inférences : quelques remarques sur la pertinence de ce concept dans les analyses psychosociales", Les Cahiers Internationaux de Psychologie Sociale, pp. 9-26.

[5] FAMA E., 1998, Market Efficiency, Long-Term Returns and Behavioural Finance, Journal of Financial Economics, 49, 3, pp283-306.

[6] FAMA, E., (1991), «Efficient Capital Markets: II », The Journal of Finance, pp. 1575-1617

[7] FAMA, E. (1965), « The behavior of stock-market prices », The Journal of Business, Vol. 38, No. 1 (Jan. 1965), pp. 34-105.

[8] GERVAIS S., ODEAN T., (2001), "Learning to be overconfident". Review of Financial Studies, 14, pp. 1-27

[9] OSBORNE M., (1962), "Periodic Structure in the Brownian Motion of Stock Prices". Operations Research, 10, pp. 345-379.

[10] RAHAOUI, R., (2007), "L'efficience informationnelle du marché financier Marocain : L'impact d'un profit warning sur les cours boursiers », Mémoire Online, http://www.memoireonline.com/02/09/1969/m_Lefficience-informationnelle-dumarchefinancier-Marocain-Limpact-dun-profit-warning-sur-les-cou10.html (consulté le 04/04/2021).

[11] ROZEFF M.S., KINNEY W.R., (1976), "Capital Market Seasonality: The Case of Stock Returns". Journal of Financial Economics, 3, pp. 379-402.

[12] SAID AIT DANI \& BOUCHRA RADI, La controverse sur l'efficience des marchés financiers et l'émergence du paradigme comportemental, Moroccan journal of business studies Vol1 Issue ${ }^{\circ} 1, \mathrm{p} 154$.

[13] SHILLER R. J., (1981),"Do stock prices move too much to be justified by subsequent changes in dividends?". American Economic Review, 71, pp. 421-498.

[14] SIMON, H.A., (1987), « Making management decisions: The role of intuition and emotion », The Academy of Management Executive, (1987-1989), Vol. 1, No. 1 (Feb., 1987), pp. 57-64.

[15] THAMI KABBAJ ,2007 'investir sans criser, bourse : saisir les opportunités " Éditions d'Organisation, pp :21-23. 
[16] TVERSKY A., KAHNEMAN D., (1974), "Judgment under uncertainty: Heuristics and biases". Science, 185, pp. 1124-1131.

[17] VENEZIA I., SHAPIRA Z., (2007), "On the behavioral differences between professional and amateur investors after the weekend". Journal of Banking \& Finance, 31, pp. 1417- 1426.

[18] WALliSER, B., (1982), "Equilibres et anticipations", Revue Economique, Vol. 33, No. 4 (Jul., 1982), pp. 594-638

[19] YANNICK COULON, « Guide pratique de la finance comportementale », Gualino éditeur, Lextenso éditions 2015, p 14. 